\title{
Cigarette Smoking and Quitting-Related Factors Among US Adult Health Center Patients with Serious Mental Illness
}

\author{
Sara Kalkhoran, MD, MAS ${ }^{1,2}$, Anne N. Thorndike, MD, MPH ${ }^{1,2}$, Nancy A. Rigotti, MD ${ }^{1,2,3}$, \\ Vicki Fung, $P h D^{2,3}$, and Travis $P$. Baggett, $M D, M P H^{1,2,4}$
}

\begin{abstract}
${ }^{1} T o b a c c o$ Research and Treatment Center, General Medicine Division, Department of Medicine, Massachusetts General Hospital, Boston, MA, USA; ${ }^{2}$ Harvard Medical School, Boston, MA, USA; ${ }^{3}$ Mongan Institute, Massachusetts General Hospital, Boston, MA, USA; ${ }^{4}$ Institute for Research, Quality, and Policy in Homeless Health Care, Boston Health Care for the Homeless Program, Boston, MA, USA.
\end{abstract}

INTRODUCTION: US adults with serious mental illness (SMI), compared to those without SMI, have a higher prevalence of smoking, which contributes to a shorter life expectancy. This study compared current smoking and quitting-related characteristics of low-income US adults with and without SMI who received healthcare at federally funded health centers.

METHODS: Using cross-sectional data from adults $\geq$ 18 years old in the nationally representative 2014 Health Center Patient Survey $(n=5592)$, we compared the prevalence of ever and current smoking among adults with and without SMI and calculated quit ratios as the percentage of ever smokers who have quit smoking. We examined the association between SMI and receiving advice to quit, making quit attempts, and having plans to quit in the next 30 days using multivariable logistic regression.

RESULTS: A total of 1376 (23\%) of participants had SMI. Ever smoking prevalence was 68\% in adults with SMI and $41 \%$ in adults without SMI, and current smoking prevalence was $48 \%$ and $22 \%$, respectively. The quit ratio was $30 \%$ and $46 \%$ among participants with and without SMI, respectively. Compared to smokers without SMI, more smokers with SMI reported receiving advice to quit in the past 12 months (aOR 2.47, 95\% CI 1.20-5.07). Smokers with and without SMI did not differ significantly in their odds of having made a past-12-month quit attempt or plans to quit.

CONCLUSIONS: Smokers with SMI seen in federally funded health centers were just as likely to have made a quit attempt and to have plans to quit as smokers without SMI. Despite a higher likelihood of receiving clinician advice to quit, the lower quit ratio in this population suggests that advice alone is unlikely to be sufficient. These results underscore the need for augmented strategies to promote smoking cessation and reduce the excess burden of tobacco-related disease in patients with SMI.

KEY WORDS: smoking cessation; mental health; vulnerable populations.

J Gen Intern Med 34(6):986-91

DOI: $10.1007 / \mathrm{s} 11606-019-04857-3$

(C) Society of General Internal Medicine 2019

Received June 4, 2018

Revised December 13, 2018

Accepted January 10, 2019

Published online February 19, 2019

\section{INTRODUCTION}

The prevalence of smoking among US adults with serious mental illness (SMI) is two- to threefold higher than in the general population, ${ }^{1}$ contributing to a shorter life expectancy than individuals without SMI. ${ }^{2,3}$ Individuals with mental illness have a mortality rate that is over twice as high as those without mental illness or the general population. ${ }^{3-5}$ Recent evidence suggests that tobacco-related conditions account for approximately half of deaths among individuals with schizophrenia, bipolar disorder, and depression, ${ }^{6}$ underscoring the importance of understanding the factors associated with smoking and quitting among people with SMI.

While smoking rates have been declining among adults in the USA, the reduction has been significantly less among those with mental illness. ${ }^{7}$ In 2016, the prevalence of smoking among all US adults was $15.5 \%{ }^{1}$ this is in contrast to estimates that over a third of individuals with bipolar disorder and over $60 \%$ of individuals with schizophrenia smoke. ${ }^{8}$ Smokers with SMI have lower cessation rates ${ }^{9,10}$ and face a number of challenges to quitting, ${ }^{11}$ such as being heavier smokers ${ }^{12}$ and experiencing more nicotine withdrawal. ${ }^{13}$ Other barriers include beliefs among healthcare providers that patients with mental illness are not interested in quitting or that quitting smoking would negatively impact mental health. ${ }^{14}$ However, studies have found that motivations to quit smoking are similar among smokers with SMI and the general population, ${ }^{15}$ and that smoking cessation is associated with improvement in psychiatric symptoms rather than worsening. ${ }^{16}$

Despite increasing recognition that smoking cessation treatment should be a key component of the care of individuals with SMI, little is known about smoking, quitting-related behaviors, and advice to quit among patients with SMI seen in safety net clinic settings. Federally funded health centers serve underserved and vulnerable patients throughout the USA. Both mental health diagnoses and tobacco use are common in patients receiving care in health centers. A prior study found that $50 \%$ of adult health center patients had a lifetime history of depression, $12 \%$ had current SMI, and $21 \%$ had accessed mental health services in the preceding year. ${ }^{17}$ Cigarette smoking is also prevalent among patients seen at adult health centers, with $33 \%$ reporting current smoking in 
2009. ${ }^{18}$ In the present study, we analyzed a survey of a nationally representative sample of patients seen in federally funded health centers to determine the association between SMI and smoking, receipt of clinician advice to quit, and quitting-related interest and behaviors among adults who received care at these sites in 2014-2015.

\section{METHODS}

\section{Data Source and Study Population}

We used cross-sectional data from the 2014 Health Center Patient Survey (HCPS), a nationally representative survey of patients receiving care at any of four types of health centers funded by the Health Resources and Services Administration Bureau of Primary Health Care grant programs (Community Health Center Program, Migrant Health Center Program, Health Care for the Homeless Program, and Public Housing Primary Care Program), with each serving specific underserved populations within the 50 US states and District of Columbia. ${ }^{19}$ Data collection occurred between September 2014 and April 2015, and patients had to have received services at the health center in the preceding 12 months to be eligible for the survey. Surveys were administered in-person using computer-assisted personal interviewing in one of five languages: English, Spanish, Chinese, Korean, or Vietnamese. Participants were compensated $\$ 25$ for participation. Of eligible individuals, $91.4 \%$ participated in the interviews.

We used publicly available data from adults $\geq 18$ years old in the HCPS. This project was deemed exempt by the institutional review board at Partners Healthcare.

\section{Measures}

Serious Mental Illness. We defined SMI as either (1) a score $\geq$ 13 on the Kessler 6 (K6) scale $e^{20,21}$ or (2) reporting being told by a health professional of a diagnosis of bipolar disorder or schizophrenia. The $\mathrm{K} 6$ measures how often in the past 30 days participants had felt each of the following symptoms: (1) so sad that nothing could cheer you up, (2) nervous, (3) restless or fidgety, (4) hopeless, (5) that everything was an effort, or (6) worthless. Each response was provided on a scale from "none of the time" (coded as 0 ) to "all of the time" (coded as 4), with a total score range from 0 to 24 . The $\mathrm{K} 6$ has been validated and used in numerous clinical and population-based contexts and countries. ${ }^{20,22,23}$ While the K6 does not identify specific psychiatric diagnoses, it allows for the identification of individuals with any mental illness that results in major impairment. Prior studies have shown that a K6 cutpoint of 13 is highly specific for identifying SMI and K6's ability to detect psychiatric disorders. ${ }^{20}$

Smoking-Related Variables. We defined ever smokers as those who reported having smoked at least 100 cigarettes in their lifetime, and never smokers as those who did not smoke 100 cigarettes in their lifetime. We further characterized ever smokers as current smokers (those who reported currently smoking either "every day" or "some days") or former smokers (those who reported now smoking "not at all").

The quit ratio is the percentage of ever smokers who have quit smoking at a point in time. ${ }^{1}$ The quit ratio was determined as the ratio of former smokers to ever smokers.

A past-12-month quit attempt was defined as having stopped smoking for more than 1 day to try to quit smoking. Participants also reported whether a doctor or other health professional advised them to quit cigarettes or other tobacco products in the preceding 12 months. Smokers who reported having plans to quit cigarettes for good in the future and had a time frame in mind for quitting were asked whether they planned to quit in the next 7 days, 30 days, 6 months, year, or more than 1 year (dichotomized into within 30 days and not within 30 days).

Covariates. Social and demographic factors in analyses included age (18-34, 35-54, $\geq 55$ ), sex, race/ethnicity (nonHispanic white, non-Hispanic black, non-Hispanic other [including Asian], Hispanic), education (less than high school, high school, more than high school), income as a percentage of the federal poverty level, and being uninsured for six or more months in the past year. We also controlled for health center clinic type. Medical comorbidities were measured as reporting being told by a doctor or other health professional of a diagnosis of asthma, chronic obstructive pulmonary disease (defined as COPD, emphysema, or chronic bronchitis), hypertension, hyperlipidemia, diabetes, cardiovascular disease (defined as coronary heart disease, angina, heart attack, or stroke), or cancer; these were categorized into 0,1 , or 2 or more. Ever use of drugs was defined as lifetime use of any of the following: marijuana (specifically non-medical use), cocaine, amphetamine-type stimulants, non-medical use of sedatives or sleeping pills, or non-medical use of opiates. Symptoms of alcohol use disorder were derived from the Alcohol, Smoking and Substance Involvement Screening Test, ${ }^{24}$ and were defined as having any of the following symptoms related to alcohol in the past 3 months: strong desire or urge to use; health, social, legal, or financial problems; failure to do what was normally expected; concern about use of alcoholic beverages from a friend or relative or anyone else; or trying and failing to control, cut down, or stop using alcohol beverages. We also controlled for being a daily smoker in analyses because the smoking cessation behaviors of daily smokers differ from non-daily smokers. ${ }^{25}$

Statistical Analysis. The HCPS included a total of 7002 participants (adults and children), of whom 5592 were adults aged 18 and older. Our study sample included adults who responded to the questions about the presence or absence of SMI $(n=5561)$. We first compared adults with and without SMI using chi-squared tests and design-adjusted Wald test statistics. We determined prevalence of ever and current smoking among adults with and without SMI. We then compared 
prevalence of past-12-month quit attempts (primary outcome), past-12-month receipt of advice to quit from a health professional, and plans to quit in the next 30 days among current smokers with and without SMI. We assessed the association between having SMI and these quitting-related outcomes using multivariable logistic regression adjusting for the covariates above. We used complex survey procedures in Stata 14 (College Station, TX) for all analyses. All counts are unweighted and all percentages are weighted to reflect the population of health center patients nationally. Weights reflect probability of selection, a non-response adjustment, and a poststratification adjustment.

\section{RESULTS}

\section{Study Sample Characteristics}

The overall prevalence of SMI in the study sample was $23 \%$, and of these individuals, $13 \%$ reported schizophrenia, 50\% reported bipolar disorder, and $64 \%$ had a Kessler 6 score $\geq 13$. Among adults with a Kessler 6 score $\geq 13,29 \%$ reported a diagnosis of either bipolar disorder or schizophrenia. Compared to adults without SMI, more adults with SMI were aged 35-54, of non-Hispanic white race/ethnicity, and living under the federal poverty level (Table 1). Adults with SMI had a higher prevalence of asthma, COPD, and cardiovascular disease compared to adults without SMI. Most participants with and without SMI were seen at Community Health Centers, reflecting the distribution of health center patients nationally; however, adults with SMI were more likely to be seen at Migrant Health Centers and less likely to be seen at Community Health Centers compared to adults without SMI. Adults with SMI had higher prevalence of ever drug use and symptoms of alcohol use disorder compared to smokers without SMI (Table 1).

\section{Smoking Status}

The prevalence of ever smoking was $68 \%$ in adults with SMI and $41 \%$ in adults without SMI $(p<0.001)$. Current smoking was also significantly higher among those with SMI (Table 2). The quit ratio for participants with SMI was significantly lower than that for those without SMI (Table 2).

In adjusted analyses, adults with SMI had nearly twice the odds of current smoking compared to adults without SMI (aOR 1.93, 95\% confidence interval (CI) 1.30-2.86; data not shown in tables).

\section{Quitting-Related Factors}

SMI was non-significantly negatively associated with being a former smoker among ever smokers ( $p=0.05$, Table 3$)$. Compared to smokers without SMI, smokers with SMI had approximately 2.5 times the odds of reporting receipt of advice to quit from a doctor or health professional in the past 12 months in adjusted analyses (Table 3). Smokers with SMI had higher odds of making a quit attempt in the past 12 months compared to smokers without SMI in unadjusted analyses, but this was not statistically significant in adjusted analyses. Smokers with and without SMI did not differ significantly in their intentions to quit in the next 30 days in unadjusted and adjusted analyses (Table 3).

\section{DISCUSSION}

In this national study of health center patients, over $80 \%$ of smokers with SMI reported receiving advice to quit smoking from a healthcare professional. Smokers with SMI reported a similar likelihood of attempts at and interest in quitting compared to smokers without SMI. These findings are concordant with other studies ${ }^{26}$ in dispelling the notion that smokers with SMI are uninterested in quitting. Despite a higher likelihood of receiving clinician advice to quit, the low quit ratio in this population suggests that advice alone is unlikely to be sufficient in this population and underscores the need for augmented strategies to promote smoking cessation and reduce the burden of tobacco-related disease in this vulnerable group of people.

Most health center smokers with SMI (87\%) reported being advised to quit smoking by a healthcare professional and $17 \%$ reported plans to quit smoking in the next 30 days. It is not clear whether participants received assistance in quitting smoking, or whether they had follow-up arranged to assess progress with smoking cessation. Future iterations of the Health Center Patient Survey should consider collecting this information to find gaps in treatment provision. To help patients with SMI translate this advice into action, health center clinicians should provide their patients who smoke with evidence-based smoking cessation treatment. Although we were not able to assess whether smokers were provided with such treatments, studies in other settings and populations have found that many patients who are screened for tobacco use and provided with advice to quit do not receive assistance in their quit attempts. ${ }^{27-29}$ The same evidence-based smoking cessation treatments that are used in the general population, ${ }^{30}$ e.g., nicotine replacement therapy, varenicline, and bupropion, have demonstrated efficacy and tolerability in populations with mental illness, including SMI. ${ }^{31-33}$ There is also evidence that extending varenicline treatment after 12 weeks to 1 year in smokers with schizophrenia and bipolar disorder improves long-term cigarette abstinence rates, ${ }^{34}$ a strategy that may be considered to improve cessation rates.

We were unable to distinguish whether participants discussed smoking with their primary care provider or psychiatrist, or both. An online survey of patients with bipolar disorder found that only one-third were advised to quit smoking by a mental health provider, ${ }^{26}$ which is much lower than the $87 \%$ reporting advice to quit from a healthcare professional in the present study. Differences in survey administration (online vs among those who had visited a health center), study 
Table 1 Sociodemographic Characteristics of Population by the Presence of Serious Mental Illness (SMI)

\begin{tabular}{|c|c|c|c|c|c|c|c|}
\hline & \multicolumn{3}{|c|}{ SMI $(n=1376)$} & \multicolumn{3}{|c|}{ No SMI $(n=4185)$} & \multirow[t]{2}{*}{$P$ value } \\
\hline & $N$ & Weighted \% & $95 \% \mathrm{CI}$ & $N$ & Weighted \% & $95 \% \mathrm{CI}$ & \\
\hline \multicolumn{8}{|l|}{ Age } \\
\hline $18-34$ & 272 & 30 & $24-37$ & 1059 & 36 & $31-42$ & \multirow{3}{*}{0.001} \\
\hline $35-54$ & 707 & 49 & $42-55$ & 1665 & 35 & $30-39$ & \\
\hline$>55$ & 397 & 21 & $17-26$ & 1461 & 29 & $23-36$ & \\
\hline Fēmale sex & 832 & 70 & $63-76$ & 2671 & 63 & $58-67$ & 0.09 \\
\hline \multicolumn{8}{|l|}{ Race/ethnicity } \\
\hline Non-Hispanic white & 455 & 57 & $47-66$ & 892 & 47 & $40-54$ & \multirow[t]{4}{*}{0.008} \\
\hline Non-Hispanic black & 344 & 19 & $14-25$ & 920 & 19 & $14-24$ & \\
\hline Non-Hispanic other & 173 & 8 & $5-13$ & 668 & 7 & $4-10$ & \\
\hline Hispanic & 403 & 16 & $12-21$ & 1703 & 27 & $22-34$ & \\
\hline \multicolumn{8}{|l|}{ Education } \\
\hline Less than HS & 616 & 34 & $28-41$ & 1813 & 36 & $32-40$ & \multirow[t]{3}{*}{0.89} \\
\hline HS & 387 & 30 & $24-35$ & 1122 & 29 & $24-34$ & \\
\hline More than HS & 371 & 36 & $30-43$ & 1236 & 36 & $32-39$ & \\
\hline Uninsured for 6 or more months in the past year & 375 & 29 & $20-41$ & 1272 & 32 & $26-38$ & 0.66 \\
\hline Income less than federal poverty level & 1068 & 71 & $64-77$ & 2534 & 52 & $47-56$ & $<0.001$ \\
\hline \multicolumn{8}{|l|}{ Clinic type } \\
\hline Public Housing Primary Care & 111 & 2 & $1-3$ & 334 & 1 & $1-2$ & \multirow[t]{4}{*}{$<0.001$} \\
\hline Health Care for the Homeless & 115 & 2 & $1-3$ & 687 & 4 & $3-5$ & \\
\hline Migrant Health Center & 495 & 8 & $6-12$ & 662 & 3 & $2-5$ & \\
\hline Community Health Center & 655 & 88 & $85-91$ & 2502 & 92 & $90-94$ & \\
\hline \multicolumn{8}{|l|}{ Medical comorbidities } \\
\hline Asthma & 341 & 23 & $19-27$ & 569 & 14 & $11-16$ & $<0.001$ \\
\hline COPD & 231 & 15 & $11-21$ & 245 & 5 & $4-7$ & $<0.001$ \\
\hline Hypertension & 755 & 48 & $41-55$ & 1868 & 44 & $39-49$ & 0.40 \\
\hline Hyperlipidemia & 586 & 44 & $37-52$ & 1447 & 42 & $38-45$ & 0.58 \\
\hline Diabetes & 306 & 24 & $20-30$ & 966 & 22 & $19-26$ & 0.41 \\
\hline Cardiovascular disease & 255 & 19 & $14-25$ & 407 & 10 & $8-13$ & 0.003 \\
\hline Cancer & 130 & 7 & $5-10$ & 250 & 6 & $4-8$ & 0.41 \\
\hline Ever drug use & 857 & 66 & $58-73$ & 1593 & 41 & $35-47$ & $<0.001$ \\
\hline Symptoms of alcohol use disorder & 409 & 28 & $23-34$ & 752 & 17 & $14-21$ & $<0.001$ \\
\hline
\end{tabular}

All N's are unweighted and all percentages are population-weighted

HS high school, COPD chronic obstructive pulmonary disease

population (bipolar disorder vs SMI), and years of data collection (2007-2008 vs 2014) may be some contributing factors. Further research should explore the extent to which smoking cessation interventions are occurring in various clinical settings. Nevertheless, all providers who see patients with SMI-including psychiatrists and primary care providers - should provide evidence-based smoking cessation treatments to smokers with SMI during all clinical encounters to maximize the likelihood that they receive treatment and support. For trainees, tobacco treatment curricula during residency programs can improve knowledge and confidence in treating patients who smoke. ${ }^{35}$ Academic detailing through strategies such as videoconference can be used to educate providers on smoking cessation treatment options and use. ${ }^{36}$

Nearly half of all adult health center patients with SMI in our study reported current cigarette use, and SMI was an independent predictor of current smoking. The 2009 Health Center Patient Survey similarly found that $56 \%$ of adults with severe mental illness as defined by a Kessler 6 score of $\geq 13$ reported current smoking, ${ }^{18}$ and overall, these prevalence rates are consistent with those from other nationally representative $^{12}$ and non-nationally representative samples of adults with SMI. ${ }^{8,37}$ Despite these higher smoking rates and the lower quit ratio among individuals with SMI compared to those without SMI, over half of smokers with SMI reported trying to quit in the past year. Thus, a major challenge remains in how to translate motivations to quit into cessation among smokers with SMI. Smokers with SMI cite various barriers to smoking cessation, ${ }^{38}$ including smoking to help deal with mental health symptoms and stress, and social factors such as the influences of peers or significant others. ${ }^{39}$ Smokers with low socioeconomic status also face many of the same barriers, ${ }^{38}$ and these

Table 2 Smoking Characteristics of Population by the Presence of Serious Mental Illness (SMI)

\begin{tabular}{|c|c|c|c|c|c|}
\hline & \multicolumn{2}{|l|}{ SMI $(n=1376)$} & \multicolumn{2}{|c|}{ No SMI $(n=4185)$} & \multirow[t]{2}{*}{$P$ value } \\
\hline & $N$ (unweighted) & Weighted \% (95\% CI) & $P$ value & Weighted \% (95\% CI) & \\
\hline \multicolumn{6}{|l|}{ Smoking status } \\
\hline Current smoker & 693 & $48(40-56)$ & 1025 & $22(18-28)$ & $<0.001$ \\
\hline Former smoker & 232 & $20(16-25)$ & 743 & $19(16-22)$ & \\
\hline Never smoker & 448 & $32(25-40)$ & 2414 & $59(4-63)$ & \\
\hline Quit ratio* (among ever smokers) & - & $30(23-37)$ & - & $46(38-54)$ & 0.001 \\
\hline
\end{tabular}

*Defined as the ratio of former smokers to ever smokers 
Table 3 Quitting-Related Characteristics of Ever and Current Smokers by the Presence of Serious Mental Illness (SMI)

\begin{tabular}{|c|c|c|c|c|}
\hline & \multicolumn{2}{|c|}{ No. (weighted \%) } & \multirow[t]{2}{*}{ Unadjusted OR (95\% CI) } & \multirow[t]{2}{*}{ aOR $(95 \%$ CI)* } \\
\hline & SMI & No SMI & & \\
\hline Being a former smoker (among ever smokers) & $232(30 \%)$ & $743(46 \%)$ & $0.50(0.33-0.76)$ & $0.68(0.46-1.003)$ \\
\hline Received advice to quit in past 12 months (among current smokers) & $555(87 \%)$ & $760(74 \%)$ & $2.30(1.20-4.42)$ & $2.47(1.20-5.07)$ \\
\hline Past-12-month quit attempt (among current smokers) & $372(59 \%)$ & $555(48 \%)$ & $1.56(1.01-2.40)$ & $1.47(0.93-2.30)$ \\
\hline Plans to quit in next 30 days (among current smokers) & $117(17 \%)$ & $169(17 \%)$ & $0.96(0.44-2.11)$ & $1.19(0.51-2.77)$ \\
\hline
\end{tabular}

Italics indicates statistical significance $(p<0.05)$

*Adjusted for age, sex, racelethnicity, education, being uninsured for 6 or more months in the past year, income, clinic type, ever use of illicit drugs, symptoms of alcohol use disorder, and number of medical comorbidities. Analyses among current smokers also controlled for being a daily smoker

barriers may be particularly salient in this population of health center patients in which $71 \%$ of adults with SMI reported living below the federal poverty level. Close follow-up, in addition to interventions that integrate support for mental health symptoms and attention to social influences, may help improve success rates of smoking cessation interventions and reduce relapse in this population that is motivated to quit. Furthermore, providers can emphasize to patients who are concerned about their mental health during tobacco cessation treatment that many patients' symptoms actually improve after quitting. ${ }^{16}$

Similar to other populations of individuals with low socioeconomic status ${ }^{40}$ and $\mathrm{SMI},{ }^{41}$ we found that health center patients with SMI had higher prevalence of tobacco-related diseases such as chronic obstructive pulmonary disease and cardiovascular disease. In addition to smoking, individuals with SMI also have higher prevalence of other factors that can influence cardiovascular risk and mortality, such as substance use ${ }^{42,43}$ and obesity. ${ }^{44,45}$ Multidisciplinary integrated care models are one mechanism for improving monitoring and management of co-occurring mental illness, health risk behaviors, and medical comorbidities. Through such models, mental health and primary care providers can work together toward a joint goal of improving the health of patients with SMI. Safety net health centers, which often provide both primary care and behavioral health services, are a particularly promising setting for implementing such models.

This study has several limitations. First, all data, including data on diagnoses of schizophrenia and bipolar disorder, were provided by participant self-report and are subject to reporting error, particularly if patients with SMI are reluctant to disclose diagnoses or symptoms. Second, our definition of SMI was based on both diagnoses and the Kessler 6 score and may represent a heterogenous group in terms of mental health status. However, a sensitivity analysis limiting the population to adults with bipolar disorder or schizophrenia yielded similar findings. Third, data on past-12-month quit attempts were only collected from current smokers and therefore represent failed quit attempts; we do not have estimates on the percentage of individuals who successfully quit smoking during the past 12 months. Without this information, it is possible that the higher prevalence of past-year quit attempts among those with SMI reflects a greater likelihood of failure when attempting to quit. Fourth, the quit ratio does not reflect the extent of relapse or the duration of cigarette abstinence in this population, and the quit ratio may change based on these factors over time. Finally, the study population of the Health Center Patient Survey represents individuals engaged in healthcare, who may be more motivated to change their health risk behaviors than individuals with no access to care or suboptimal visit attendance, which is more prevalent among those with SMI. ${ }^{46}$ While our findings are representative of health center patients nationally, these findings are heavily weighted by those seen in CHCs, who comprise more than $90 \%$ of patients seen in federally funded health centers each year. Results may not be generalizable to other populations of underserved adults with SMI.

Adult health center patients with SMI have a greater burden of cigarette smoking and a lower quit ratio than adults without SMI. Contrary to many clinicians' perceptions that smokers with SMI do not want to quit, over half of smokers with SMI made a quit attempt in the past year and their intentions to quit were no different from those of smokers without SMI. Providers caring for these vulnerable patients can help reduce the mortality gap in patients with SMI by providing evidencebased smoking cessation treatment to help smokers succeed in their attempts to quit.

Acknowledgments: This work was supported by the National Heart, Lung, and Blood Institute (K23HL136854, RO1HL125486), the National Institute on Drug Abuse (K23DA034008), National Institute of Diabetes and Digestive and Kidney Diseases (R01DK114735), Agency for Healthcare Research and Quality (R01HSO25378), and by a Massachusetts General Hospital Transformative Scholars Program Award.

Corresponding Author: Sara Kalkhoran, MD, MAS; Tobacco Research and Treatment Center, General Medicine Division, Department of Medicine Massachusetts General Hospital, Boston, MA, USA (e-mail: skalkhoran@mgh.harvard.edu).

\section{Compliance with Ethical Standards:}

Disclaimer: The funding sources had no role in study design; collection, analysis and interpretation of data; writing the report; or the decision to submit the report for publication. An abstract of this work was presented at the 2018 Society of General Internal Medicine Annual Meeting.

Conflict of Interest: Drs. Baggett, Kalkhoran, and Rigotti receive royalties from UpToDate. Dr. Rigotti has received a research grant from and been an unpaid consultant regarding smoking cessation to Pfizer, Inc., and a paid consultant to Achieve Life Sciences. No other financial disclosures are reported by the authors of this paper. 
Publisher's Note: Springer Nature remains neutral with regard to jurisdictional claims in published maps and institutional affiliations.

\section{REFERENCES}

1. Jamal A, Phillips E, Gentzke AS, et al. Current cigarette smoking among adults - United States, 2016. MMWR Morb Mortal Wkly Rep. 2018;67(2):53-59.

2. Tam J, Warner KE, Meza R. Smoking and the reduced life expectancy of individuals with serious mental illness. Am J Prev Med. 2016;51(6):958966.

3. Olfson M, Gerhard T, Huang C, Crystal S, Stroup TS. Premature mortality among adults with schizophrenia in the United States. JAMA Psychiatry. 2015;72(12):1172-1181.

4. Walker ER, McGee RE, Druss BG. Mortality in mental disorders and global disease burden implications: a systematic review and metaanalysis. JAMA Psychiatry. 2015;72(4):334-341.

5. Saha S, Chant D, McGrath J. A systematic review of mortality in schizophrenia: is the differential mortality gap worsening over time? Arch Gen Psychiatry. 2007;64(10):1123-1131.

6. Callaghan RC, Veldhuizen S, Jeysingh $\mathbf{T}$, et al. Patterns of tobaccorelated mortality among individuals diagnosed with schizophrenia, bipolar disorder, or depression. J Psychiatr Res. 2014;48(1):102-110.

7. BL Cook, Wayne GF, Kafali EN, Liu Z, Shu C, Flores M. Trends in smoking among adults with mental illness and association between mental health treatment and smoking cessation. JAMA. 2014;311(2):172-182.

8. Dickerson F, Schroeder J, Katsafanas E, et al. Cigarette smoking by patients with serious mental illness, 1999-2016: an increasing disparity. Psychiatr Serv. 2018;69(2):147-153.

9. Smith PH, Mazure CM, McKee SA. Smoking and mental illness in the US population. Tobacco Control. 2014;23(e2):e147-e153.

10. Streck JM, Weinberger AH, Pacek LR, Gbedemah M, Goodwin RD. Cigarette smoking quit rates among persons with serious psychological distress in the United States from 2008-2016: Are mental health disparities in cigarette use increasing? Nicotine Tob Res. 2018. https:// doi.org/10.1093/ntr/nty227.

11. Sharma R, Gartner CE, Hall WD. The challenge of reducing smoking in people with serious mental illness. Lancet Respir Med. 2016;4(10):835844 .

12. Glasheen C, Hedden SL, Forman-Hoffman VL, Colpe LJ. Cigarette smoking behaviors among adults with serious mental illness in a nationally representative sample. Ann Epidemiol. 2014;24(10):776-780.

13. Smith PH, Homish GG, Giovino GA, Kozlowski LT. Cigarette smoking and mental illness: a study of nicotine withdrawal. Am J Public Health. 2014;104(2):e127-133.

14. Sheals K, Tombor I, McNeill A, Shahab L. A mixed-method systematic review and meta-analysis of mental health professionals' attitudes toward smoking and smoking cessation among people with mental illnesses. Addiction. 2016;111(9):1536-1553.

15. Siru R, Hulse GK, Tait RJ. Assessing motivation to quit smoking in people with mental illness: a review. Addiction. 2009;104(5):719-733.

16. Taylor G, McNeill A, Girling A, Farley A, Lindson-Hawley N, Aveyard P. Change in mental health after smoking cessation: systematic review and meta-analysis. BMJ. 2014;348:g1151.

17. Jones E, Lebrun-Harris LA, Sripipatana A, Ngo-Metzger Q. Access to mental health services among patients at health centers and factors associated with unmet needs. J Health Care Poor Underserved. 2014;25(1):425-436.

18. Lebrun-Harris LA, Fiore MC, Tomoyasu N, Ngo-Metzger Q. Cigarette smoking, desire to quit, and tobacco-related counseling among patients at adult health centers. Am J Public Health. 2015;105(1):180-188.

19. Health Center Patient Survey. https://bphc.hrsa.gov/datareporting/research/hcpsurvey/index.html. Accessed April 4, 2018.

20. Kessler RC, Barker PR, Colpe LJ, et al. Screening for serious mental illness in the general population. Arch Gen Psychiatry. 2003;60(2):184189.

21. Kessler RC, Andrews G, Colpe LJ, et al. Short screening scales to monitor population prevalences and trends in non-specific psychological distress. Psychol Med. 2002;32(6):959-976.

22. Furukawa TA, Kawakami N, Saitoh M, et al. The performance of the Japanese version of the K6 and K10 in the World Mental Health Survey Japan. Int J Methods Psychiatr Res. 2008;17(3):152-158.

23. Furukawa TA, Kessler RC, Slade T, Andrews G. The performance of the $\mathrm{K} 6$ and K10 screening scales for psychological distress in the Australian
National Survey of Mental Health and Well-Being. Psychol Med. 2003;33(2):357-362.

24. The Alcohol, Smoking and Substance Involvement Screening Test (ASSIST): development, reliability and feasibility. Addiction. 2002;97(9):1183-1194.

25. Tindle HA, Shiffman S. Smoking cessation behavior among intermittent smokers versus daily smokers. Am J Public Health. 2011;101(7):e1-e3.

26. Prochaska JJ, Reyes RS, Schroeder SA, Daniels AS, Doederlein A Bergeson B. An online survey of tobacco use, intentions to quit, and cessation strategies among people living with bipolar disorder. Bipolar Disord. 2011;13(5-6):466-473.

27. Jamal A, Dube SR, Malarcher AM, Shaw L, Engstrom MC. Tobacco use screening and counseling during physician office visits among adultsNational Ambulatory Medical Care Survey and National Health Interview Survey, United States, 2005-2009. MMWR Suppl. 2012;61(2):38-45.

28. Park ER, Gareen IF, Japuntich S, et al. Primary Care Provider-Delivered Smoking Cessation Interventions and Smoking Cessation Among Participants in the National Lung Screening Trial. JAMA Intern Med. 2015;175(9):1509-1516.

29. Thorndike AN, Stafford RS, Rigotti NA. US physicians' treatment of smoking in outpatients with psychiatric diagnoses. Nicotine Tob Res. 2001;3(1):85-91.

30. Fiore MC, Jaen CR, Baker T, et al. Treating tobacco use and dependence: 2008 update. Rockville, MD: US Department of Health and Human Services. 2008.

31. Roberts E, Eden Evins A, McNeill A, Robson D. Efficacy and tolerability of pharmacotherapy for smoking cessation in adults with serious mental illness: a systematic review and network meta-analysis. Addiction. 2016;111(4):599-612.

32. Tsoi DT, Porwal M, Webster AC. Interventions for smoking cessation and reduction in individuals with schizophrenia. Cochrane Database Syst Rev. 2013(2):Cd007253.

33. Anthenelli RM, Benowitz NL, West R, et al. Neuropsychiatric safety and efficacy of varenicline, bupropion, and nicotine patch in smokers with and without psychiatric disorders (EAGLES): a double-blind, randomised, placebo-controlled clinical trial. Lancet. 2016;387(10037):25072520.

34. Prochaska JJ, Fromont SC, Leek D, et al. Evaluation of an evidencebased tobacco treatment curriculum for psychiatry residency training programs. Acad Psychiatry. 2008;32(6):484-492.

35. Prochaska JJ, Fromont SC, Leek D, et al. Evaluation of an evidencebased tobacco treatment curriculum for psychiatry residency training programs. Acad Psychiatry. 2008;32(6):484-492.

36. Brunette MF, Dzebisashvili N, Xie H, Akerman S, Ferron JC, Bartels S. Expanding cessation pharmacotherapy via videoconference educational outreach to prescribers. Nicotine Tob Res. 2015;17(8):960-967.

37. Swarbrick MA, Cook JA, Razzano LA, et al. Correlates of current smoking among adults served by the public mental health system. J Dual Diagn. 2017;13(2):82-90.

38. Twyman L, Bonevski B, Paul C, Bryant J. Perceived barriers to smoking cessation in selected vulnerable groups: a systematic review of the qualitative and quantitative literature. BMJ Open. 2014;4(12):e006414.

39. Trainor K, Leavey G. Barriers and facilitators to smoking cessation among people with severe mental illness: a critical appraisal of qualitative studies. Nicotine Tob Res. 2017;19(1):14-23.

40. Prescott E, Vestbo J. Socioeconomic status and chronic obstructive pulmonary disease. Thorax. 1999;54(8):737-741.

41. Himelhoch S, Lehman A, Kreyenbuhl J, Daumit G, Brown C, Dixon L. Prevalence of chronic obstructive pulmonary disease among those with serious mental illness. Am J Psychiatry. 2004;161(12):2317-2319.

42. RachBeisel J, Scott J, Dixon L. Co-occurring severe mental illness and substance use disorders: a review of recent research. Psychiatr Serv. 1999;50(11):1427-1434.

43. Hartz SM, Pato CN, Medeiros H, et al. Comorbidity of severe psychotic disorders with measures of substance use. JAMA Psychiatry. 2014;71(3):248-254

44. Allison DB, Newcomer JW, Dunn AL, et al. Obesity among those with mental disorders: a National Institute of Mental Health meeting report. Am J Prev Med. 2009;36(4):341-350.

45. Dickerson FB, Brown CH, Kreyenbuhl JA, et al. Obesity among individuals with serious mental illness. Acta Psychiatr Scand. 2006;113(4):306-313.

46. Lawrence D, Kisely S. Inequalities in healthcare provision for people with severe mental illness. J Psychopharmacol. 2010;24(4_supplement):61-68. 